Synthesis and analysis of 4-(3-fluoropropyl)-glutamic acid stereoisomers to determine the stereochemical purity of (4S)-4-(3-[18F]fluoropropyl)-L-glutamic acid ([18F]FSPG) for clinical use

\title{
Point-by-point Response to the Comments
}

\section{Editor Comments:}

1: Please add a statement about the use of $\mathrm{CuSO} 4$ at $2 \mathrm{mM}$. Why specifically at this concentration? Response: Thanks! The $\mathrm{CuSO}_{4}$ concentration was directly chosen based on the application example for the analysis of aspartic acid, which also possesses an acidic side-chain, in the column technical guide. A statement has been added to the text as suggested. Page 29, line 627-629.

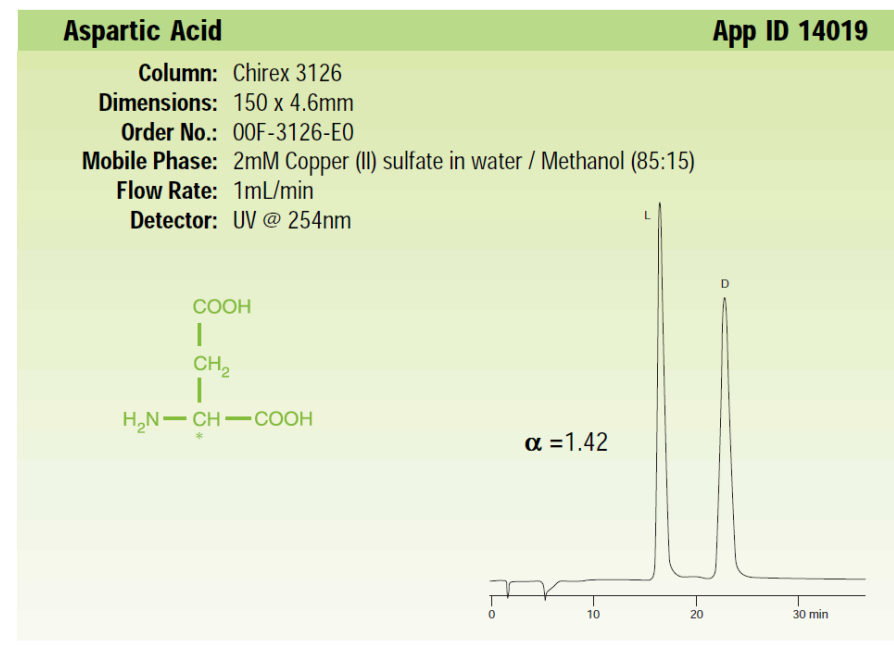

2: Most peaks in Figs S13, S15, S17 are difficult to observe, please provide an enlarge image to show those peaks with clear splitting patterns.

Response: Thanks! These figures have been enlarged as shown in Figs S13, S15, and S17.

3: $13 \mathrm{C}$ of $(2 \mathrm{~S}, 4 \mathrm{R})-11$ contains too much impurities. Please update the spectrum using pure material. Response: Thanks! The spectrum has been replaced as shown in Fig 24. 